\title{
The Galactic magnetic field in the light of starburst-generated ultrahigh-energy cosmic rays
}

\author{
Jorge F. Soriano* \\ Department of Physics \& Astronomy, Lehman College, CUNY, NY 10468, USA \\ Department of Physics, Graduate Center, City University of New York, NY 10016, USA \\ E-mail: jfdezsoriano@gmail.com
}

\section{Luis A. Anchordoqui}

Department of Physics \& Astronomy, Lehman College, CUNY, NY 10468, USA

Department of Physics, Graduate Center, City University of New York, NY 10016, USA

Department of Astrophysics, American Museum of Natural History, NY 10024, USA

E-mail: luis.anchordoqui@gmail.com

\section{Diego F. Torres}

Institute of Space Sciences (ICE-CSIC), Campus UAB, Carrer de Magrans s/n, 08193

Barcelona, Spain

Institució Catalana de Recerca i Estudis Avançats (ICREA), E-08010 Barcelona, Spain

Institut d'Estudis Espacials de Catalunya (IEEC), 08034 Barcelona, Spain

E-mail: dtorres@ice.csic.es

\begin{abstract}
Auger data show evidence for a correlation between ultrahigh-energy cosmic rays (UHECRs) and nearby starburst galaxies. This intriguing correlation is consistent with data collected by the Telescope Array, which have revealed a much more pronounced directional "hot spot" in arrival directions not far from the starburst galaxy M82. In this work, we assume starbursts are sources of UHECRs and investigate the prospects to use the observed distribution of UHECR arrival directions to constrain Galactic magnetic field models. We show that if the Telescope Array hot spot indeed originates from M82, UHECR data would place a strong constraint on the coherent and turbulent components of the Galactic magnetic field.
\end{abstract}

36th International Cosmic Ray Conference - ICRC219 -

July 24 - August 1, 2019

Madison, Wisconsin, USA

\footnotetext{
*Speaker.
} 


\section{General Idea}

Magnetic fields are one of the most challenging astrophysical phenomena to measure. We do have indications that magnetic fields are everywhere in the Universe, but they are often very weak and challenging to characterize in detail. The Milky Way is host to a magnetic field on the order of $10^{-6} \mathrm{G}$, which is nearly a million times smaller than the Earth's magnetic field. We know magnetic fields exist on galaxy scales and larger in the Universe, but we do not know how they got there, neither we do completely understand their role in how the Universe has evolved. Our observational and theoretical understanding of magnetic fields in the Milky Way and of the global structure of the Galactic magnetic field (GMF) has matured over many decades [1,2], with a new-generation of more sophisticated and quantitatively-constrained models emerging in the last decade [3-9].

GMF models are constrained by:

(i) Multi-frequency radio observations of the Faraday rotation of extragalactic radio sources. The polarization plane of a linearly polarized electromagnetic wave which propagates through a magnetized plasma rotates by an angle $\psi$ proportional to the square of the wavelength $\lambda$, i.e. $\Delta \psi=\mathrm{RM} \lambda^{2}$. To determine the rotation measure RM we then require multi- or at least bi-frequency observations. The value of RM is proportional to the line-of-sight integral

$$
\mathrm{RM}=c_{1} \int_{0}^{D} d x_{3} n_{e} B_{\|},
$$

where $c_{1} \simeq 2.7 \times 10^{-23} \mathrm{rad} / \mu \mathrm{G}$ is the proportionality constant, $B_{\|}$is the the longitudinal component of the GMF, $D$ is the distance to the source, and and $n_{e}$ is the density of thermal electrons of the warm ionized medium of the Galaxy [10].

(ii) Measurements of the polarized synchrotron emission of cosmic-ray electrons. Galactic synchrotron emission sets a constraint on the GMF that is complementary to the one from RMs, because synchrotron emission depends on the transverse GMF $B_{\perp}$, weighted by the relativistic (a.k.a. cosmic-ray) electron density $n_{\mathrm{CR} e}$. The polarization state of linearly polarized light is specified by the Stokes parameters Q and U, with each proportional to the polarized intensity (PI)

$$
\mathrm{PI}_{i} \sim \int_{0}^{D} d x_{3} \varepsilon_{i j 3} n_{\mathrm{CRe}} B_{j}^{2},
$$

where $B_{i}$ with $i=(1,2)$ are the components of $B_{\perp}$, and $\varepsilon_{i j 3}$ are components of the Levi-Civita tensor $\varepsilon_{i j k}$ [11]. The orientation of $B_{\perp}$ can be inferred from the Stokes parameters Q and U. Exploiting the interconnections (1.1) and (1.2) between the GMF and the physical observables depends on our understanding of the thermal and relativistic electron distributions.

The GMF also deflects ultra-high energy cosmic rays (UHECRs). For a cosmic ray of energy $E$ and charge $Z e$, the arrival direction $\vec{\xi}$ is related to the the point of entry into the Galaxy $\vec{\zeta}$ according to $\vec{\zeta}=\vec{\xi}+\vec{\delta}$, where $\vec{\delta}$ is proportional to the line-of-sight integral

$$
\delta_{i}=c_{2} \int_{0}^{D} d x_{3} \varepsilon_{i j 3} B_{j}^{2}
$$

with $c_{2}=Z e /(E \mu \mathrm{G})$. The similarities between (1.1), (1.2), and (1.3) suggest that knowing the nuclear composition of UHECRs and each point of entry into the Galaxy, the distribution of arrival directions provides a robust constraint on the GMF. 
The current upper limit on the extragalactic magnetic field is $B \sim 1 \mathrm{nG}$ [12], and so the typical deflection from a source $3.5 \mathrm{Mpc}$ away is estimated to be $[13,14]$

$$
\delta \theta_{\mathrm{eg}} \lesssim 1.5^{\circ} Z\left(\frac{E}{10^{10} \mathrm{GeV}}\right)^{-1}
$$

For reasons outlined below, herein we are interested in UHECR nuclei of $Z \leq 8$ and $E \gtrsim 10^{10.6} \mathrm{GeV}$. For nearby sources, the expected deflections of these nuclei on the extragalactic magnetic field are $\lesssim 3^{\circ}$. This implies that the galactic longitude $l$ and latitude $b$ indicating the UHECR point of entry into the Galaxy are roughly coincident with the coordinates of the source location.

In summary, to constrain the GMF using UHECR observations we need high-resolution measurements of the arrival direction distribution and the mass spectrum, and we also need to identify the sources. To successfully fit our guides, we start out in the next section by pinpointing a possible correlation between UHECRs and starburst galaxies (SBGs), as a first step in the source identification. In the last section, we estimate the expected deflections from nearby starbursts on the basis of existing GMF models and comment on the prospects to measure the mass spectrum with future experiments.

\section{Setting the Stage}

We have long been suspecting that SBGs are sources of UHECRs [15]. Over the years, stronger and stronger experimental evidence has been accumulating indicting a possible correlation between the arrival directions of the highest energy cosmic rays and nearby SBGs [16-19].

Using data collected by the Pierre Auger Observatory, the hypothesis of UHECR emission from the 23 brightest nearby SBGs with a radio flux larger that $0.3 \mathrm{Jy}$ (selected out the $63 \mathrm{ob}-$ jects within $250 \mathrm{Mpc}$ search for $\gamma$-ray emission by the Fermi-LAT Collaboration [20]) was tested against the null hypothesis of isotropy through an unbinned maximum-likelihood analysis [19]. The adopted test statistic (TS) for deviation from isotropy being the standard likelihood ratio test between the starburst-generated UHECR sky model and the null hypothesis. The TS was maximized as a function of two free parameters (the angular radius common to all sources, which accounts in an effective way for the magnetic deflections, and the signal fraction), with the energy threshold varying in the range $10^{10.3} \lesssim E / \mathrm{GeV} \lesssim 10^{10.9}$. For a given energy threshold, the TS for isotropy follows a $\chi^{2}$ distribution with two degrees of freedom. The TS is maximum above $10^{10.6} \mathrm{GeV}$, with a local $p$-value of $3 \times 10^{-6}$. The smearing angle and the anisotropic fraction corresponding to the best-fit parameters are $13_{-3}^{+4^{\circ}}$ and $(10 \pm 4) \%$, respectively. Remarkably, the energy threshold of largest statistical significance coincides with the observed suppression in the spectrum [18], implying that when we properly account for the barriers to UHECR propagation in the form of energy loss mechanisms [21,22] we obtain a self consistent picture for the observed UHECR horizon. The scan in energy thresholds comes out with a penalty factor, which was estimated through Monte-Carlo simulations. The post-trial chance probability in an isotropic cosmic ray sky is $4.2 \times 10^{-5}$, corresponding to a 1 -sided Gaussian significance of $4 \sigma$ [19].

Very recently, the Telescope Array (TA) Collaboration carried out a test of the reported correlation between the arrival directions of UHECRs and SBGs [19]. The data are compatible with 
isotropy to within $1.1 \sigma$ and with Auger result to within $1.4 \sigma$, and so the TA Collaboration concluded that with their current statistics they cannot make a statistically significant corroboration or refutation of the reported possible correlation between UHECRs and SBGs [23]. However, TA data have revealed a pronounced directional "hot spot" [24] in arrival directions not far from the starburst galaxy M82 [25,26]. In this work we show that if the TA hot spot indeed originates from M82, UHECR data would place a strong constraint on GMF models.

The most recent search for hot spot anisotropies is a joint effort by the Auger and TA collaborations considering 840 events recorded by Auger with $E_{\text {Auger }}>10^{10.6} \mathrm{GeV}$ and 130 events recorded by TA with $E_{\mathrm{TA}}>10^{10.73} \mathrm{GeV}$ [27]. Before proceeding, we pause to note that even though the techniques for assigning energies to events are nearly the same in both experiments, there are differences as to how the primary energies are derived at Auger and TA, with systematic uncertainties in the energy scale of the experiments amounting to about $14 \%$ and $21 \%$ respectively, corresponding to about $70 \%$ uncertainty in the flux above a fixed energy threshold. By comparison, the uncertainties on the respective exposures are minor $(\lesssim 1 \%$ and $\simeq 3 \%$, respectively). Therefore, it is necessary to cross-calibrate the energy scales of the two datasets to avoid introducing a spurious North/South asymmetry due to an energy scale mismatch. This is accomplished by exploiting the wide declination band $\left(-16^{\circ} \lesssim \delta \lesssim+45^{\circ}\right)$ where the two datasets overlap. Regardless of the true arrival direction distribution, within a region of the sky $\Delta \Omega$ fully contained in the field of view (FoV) of both observatories, the sum over observed events $\sum_{i} 1 / \omega\left(\mathbf{n}_{i}\right)$ (where $\omega$ is the directional exposure of each observatory in the direction $\mathbf{n}_{i}$, in $\mathrm{km} \mathrm{yr}$ units) is an unbiased estimator of $\int_{\Delta \Omega} \Phi(\mathbf{n}) d \mathbf{n}$ (where $\Phi$ is the directional UHECR flux integrated above the considered energy threshold, in $\mathrm{km}^{-2} \mathrm{yr}^{-1} \mathrm{sr}^{-1}$ units) and should be the same for both experiments except for statistical fluctuations. This criterium is generally adopted to cross-calibrate the energy scales and to determine $E_{\text {Auger }}$ and $E_{\mathrm{TA}}$ such that the Auger flux above $E_{\text {Auger }}$ matches the TA flux above $E_{\mathrm{TA}} \cdot{ }^{1}$ The most significant excesses observed in a $20^{\circ}$ search are at: $(l, b) \approx\left(303.0^{\circ}, 12.9^{\circ}\right)$ and $(l, b) \approx\left(162.5^{\circ}, 44.4^{\circ}\right)$, with local (Li-Ma [28]) statistical significance for the rejection of the null (background only) hypothesis of $4.7 \sigma$ and $4.2 \sigma$, respectively. The Li-Ma significance map of this data-sample is shown in Fig. 1. The most significant hot spot is near the location of starburst galaxies NGC 4945 and M83. The starburst galaxy M82 is at the northern edge of the TA hot spot. A warm spot is also visible in the skymap near the direction of the closest starburst NGC 253. In closing, we note that the clear and convincing evidence for the correlation between UHECRs and SBGs is further supported by a solid framework for particle acceleration to the highest observed energies [29-31]. In our calculations we will then assume that SBGs are the sources of UHECRs.

\section{Results and Conclusions}

The global structure of the GMF can be divided into the halo and disk components. Each component can further be subdivided into a coherent regular field $B_{\text {reg }}$, which yields directional deflections and a random field $B_{\text {rand }}$. Pshirkov, Tinyakov, Kronberg and Newton-McGee (PTKN) used data from the NRAO VLA Sky Survey rotation measures catalog [35] to constrain the GMF [3].

\footnotetext{
${ }^{1}$ Actually, the region of the sky which is mostly used spans the declination band $-12^{\circ} \leq \delta \leq+42^{\circ}$. This is because including directions too close to the edge of the FoV of one of the observatories would result in larger statistical fluctuations due to very large values of $1 / \omega\left(\mathbf{n}_{\mathbf{i}}\right)$ near the edge.
} 


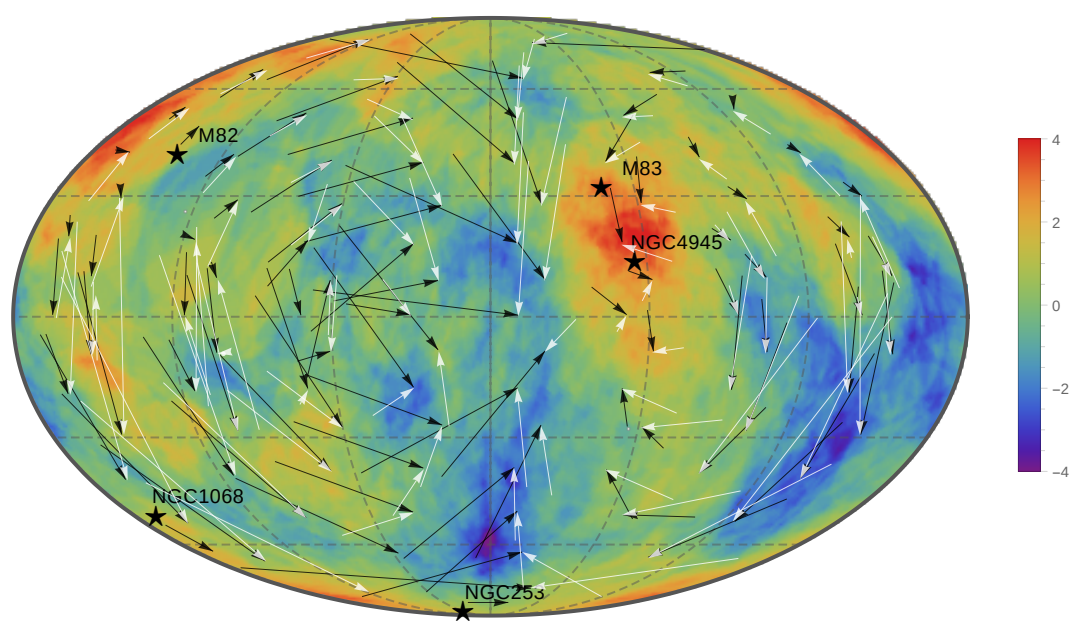

Figure 1: Skymap in Galactic coordinates of the Li-Ma significances of overdensities in $20^{\circ}$ radius windows for 840 events recorded by Auger with $E>E_{\text {Auger }}$ and 130 events recorded by TA with $E>E_{\mathrm{TA}}$. The color scale indicates the significance in units of standard deviations; negative values follow the convention of indicating the (positive) significance of deficits. We have superimposed the expected deflections from UHECR protons with $E=10^{10} \mathrm{GeV}$ as predicted by the PTKN (white) [3] and JF (black) [4,5] models [3234]. The beginning of the arrows indicate the location of the sources and the tip of the arrows indicate the arrival direction on Earth. The Galactic Center is at the center of the skymap. The RGB color components of the skymap and legend presented in [27] were sampled taking enough points per pixel to ensure that no information is loss. To each point sampled from the skymap, we associate a value for the $\mathrm{Li}$-Ma significance given by the corresponding value of the legend pixel that is closest to the skymap pixel. The closeness is measured by a euclidean distance in the RGB space. The coordinates of the pixels were transformed successively by an inverse Mollweide projection, an equatorial to galactic coordinate transformation, and a Mollweide projection to create the new skymap shown in this figure.

The observed distribution of RMs over the sky disfavors ring disk models. A spiral disk and antisymmetric halo structure best fit the data. Targeted observations of Galactic structures and high resolution synchrotron mapping of external galaxies such as in the CHANGES survey [36] as well as sky maps of polarized and unpolarized Galactic synchrotron emission from WMAP were considered by Jansson and Farrar (JF) to complement RMs and develop a sophisticated GMF model $[4,5]$. (The 7-year WMAP synchrotron maps [37] were used in the original JF analysis [4,5]; the 9-year final WMAP data release [38] and the Planck 2015 data release [39] were considered in the update of [7].) The JF model contains three distinct components: (1) a coherent large-scale field, with disk, halo and out-of-plane components, (2) a fully random field specified by its spatiallyvarying rms field strength, and (3) a "striated" random field. (1.a) The disk component of the coherent field is toroidal in the inner "molecular ring" region from $3-5 \mathrm{kpc}$, beyond which it has a logarithmic-spiral geometry. The typical strength of the coherent disk field in the magnetic arms is roughly $1 \mu \mathrm{G}$, with maximum values of a few $\mu \mathrm{G}$. (1.b) The regular halo component is modelled as oppositely directed coherent toroidal fields above and below the Galactic Plane. The sense of rotation below the plane (Southern hemisphere) is in the same direction as the rotation of the disk. The toroidal fields reach their maximum strength $\mathscr{O}(\mu \mathrm{G})$ about $1 \mathrm{kpc}$ away from the plane, beyond which they decline slowly reaching half their peak value at about $5 \mathrm{kpc}$. (1.c) The out-of- 
plane halo component of the coherent field or (X-field) is azimuthally symmetric and poloidal; its strength is $5 \mu \mathrm{G}$ at the Galactic center, diminishing rather slowly with distance from the Galactic plane. The radial-scale length of the $\mathrm{X}$-field is about $3 \mathrm{kpc}$ and its value in the solar neighborhood is approximately $0.2 \mu \mathrm{G}$. The sense of the halo toroidal fields are consistent with their resulting from differential rotation of the coherent poloidal X-field. The disk, toroidal halo, and X fields were required to be separately divergenceless, so their free parameters could be adjusted independently. (2) The random field is modelled as a superposition of a disk component whose spiral-arms are the same as those adopted for the coherent field, but with independently fitted rms field strength, and a smooth halo component. The halo field has an azimuthal component, which can be characterized by its overall rms strength, and radial and vertical scale lengths. The understanding of the random field structure and its maximum strength are muddled by the uncertainty in $n_{\mathrm{CRe}}$. The best-estimate for the maximum field strength is $\mathscr{O}(10 \mu \mathrm{G})$. (3) The striated field is aligned with the local coherent field and its rms strength is locally proportional to the coherent field strength, that is $B_{\text {stri }}^{2} \propto B_{\text {reg }}^{2}$.

Using (1.3), the expected deflections of UHECRs when crossing a distance $L$ of the Galaxy are estimated to be [32]

$$
\delta \theta_{\mathrm{G}} \simeq 10^{\circ} Z\left(\frac{E}{10^{10} \mathrm{GeV}}\right)^{-1}\left|\int_{0}^{L} \frac{d \vec{x}}{\mathrm{kpc}} \times \frac{\vec{B}}{2 \mu \mathrm{G}}\right| .
$$

This implies that particles in the energy range $10^{10.6} \lesssim E / \mathrm{GeV} \lesssim 10^{11.3}$, which would suffer deflections of $\sim 13^{\circ}$, are most likely CNO, with $Z \leq 8$. Note that the helium contribution to the flux will be largely suppressed because of energy loss during propagation [40]. In our calculations we then take as fiducial a particle rigidity of $10^{10} \mathrm{GV}$. For, $Z=1$ and $E=10 \mathrm{EeV}$ we have $c_{2}=3 \times 10^{-23} \mathrm{rad} /(\mu \mathrm{Gcm})[10]$. For further reference, $c_{1} / c_{2}=0.9 \mathrm{~cm}$. In Fig. 1 we show the expected deflection for protons of $E=10^{10} \mathrm{GeV}$ according to the PTKN [3] and JF [4,5] GMF models [32-34]. It is clear from the figure that the expected deflections are consistent (at least at the qualitative level) with the observed excess in Auger data. However, on the assumption that the TA hot spot originates in the SBG M82 we conclude that the expected deflections exhibit a poor representation of the TA data. More concretely, the expected deflections of UHECRs entering the Galaxy from the direction of M82 shown in Fig. 1 are towards the Galactic north-east of the M82, whereas the center of the TA hot spot is in the Galactic north-west direction of the source. Note that this is the case for both GMF models shown in the figure, and also for all possible variations of the JF configuration discussed in [7]. We conclude that if the starburst hypothesis is validated by future Auger data, then M82 must be a powerful source of UHECRs and must dominate the contribution to the TA hot spot. This can be used to constrain the GMF with future UHECR data, for which the nuclear composition of each event is known.

The Probe Of Extreme Multi-Messenger Astrophysics (POEMMA) is a NASA space-based mission [41]. POEMMA is optimized for the measurement of extensive air showers (EASs) from UHECRs using the stereo air fluorescence technique, and from neutrino induced upwardgoing EASs via optical Cherenkov detection. POEMMA makes observations in umbra and in low moonlight conditions. POEMMA is designed to reach unprecedented geometrical apertures $>10^{6} \mathrm{~km}^{2} \mathrm{sr} \mathrm{yr}$, which after duty cycle corrections, correspond to annual exposures of more than $10^{5} \mathrm{kmsryr}$ at the highest energies. POEMMA is composed of two identical satellites flying in 
formation with the ability to observe overlapping regions during moonless nights at angles ranging from nadir to just above the limb of the Earth. The satellites will fly at an altitude of about $525 \mathrm{~km}$, with separations ranging from $300 \mathrm{~km}$ for stereo fluorescence UHECR observations to $25 \mathrm{~km}$ when pointing at the Earth's limb for both fluorescence and Cherenkov observations of UHECRs and cosmic neutrinos. The satellites will orbit the Earth with a period of 95 minutes, orbiting the Earth $\sim 15$ times per day. POEMMA has full-sky coverage due to its orbit at $525 \mathrm{~km}$ altitude and $28.5^{\circ}$ inclination and the very large field-of-view $\left(45^{\circ}\right)$ for each satellite. The ability of the space-based POEMMA telescopes to tilt towards the northern or southern hemisphere allows for the sky exposure can be enhanced for a specific hemisphere. Likewise, it is easy for POEMMA to view north or south for a sequence of orbital periods to further tailor the UHECR sky coverage for possible source locations.

The atmospheric column depth at which the longitudinal development of an EAS reaches maximum, $X_{\max }$, is a powerful observable to determine the UHECR nuclear composition. Detailed simulations of POEMMA's UHECR exposure, angular resolution, and $X_{\max }$ resolution were performed using the instrument design [42]. POEMMA stereo observations of EASs will have high angular resolution $\lesssim 1^{\circ}$ for $E>10^{10.5} \mathrm{GeV}$. The fine angular resolution leads to accurate 3 -dimensional reconstruction of the EASs, with energy resolution of $\sim 20 \%$ and $X_{\max }$ resolution of $\sim 35\left(10^{10.6} \mathrm{GeV} / E\right)^{0.55} \mathrm{~g} / \mathrm{cm}^{2}$. The event-by-event composition measurements together with the full-sky distribution of arrival directions will provide a profitable data-sample for constraining GMF models.

In summary, the GMF has always been seen as a hindrance for charged particle astronomy. In the spirit of [43], here we have shown that now we can turn things around and use UHECR deflections to constrain GMF models.

\section{Acknowledgments}

We thank our colleagues of the POEMMA and Pierre Auger collaborations for discussion. This work has been supported by the U.S. National Science Foundation (NSF Grant PHY-1620661), the National Aeronautics and Space Administration (NASA Grant 80NSSC18K0464), as well as by grants PGC2018-095512-B-I00, AYA2017- 92402-EXP, iLink 2017-1238, and SGR 2017-1383.

\section{References}

[1] R. Beck, A. Brandenburg, D. Moss, A. Shukurov and D. Sokoloff, Ann. Rev. Astron. Astrophys. 34, 155 (1996).

[2] L. M. Widrow, Rev. Mod. Phys. 74, 775 (2002).

[3] M. S. Pshirkov, P. G. Tinyakov, P. P. Kronberg and K. J. Newton-McGee, Astrophys. J. 738, 192 (2011).

[4] R. Jansson and G. R. Farrar, Astrophys. J. 757, 14 (2012).

[5] R. Jansson and G. R. Farrar, Astrophys. J. 761, L11 (2012).

[6] M. C. Beck, A. M. Beck, R. Beck, K. Dolag, A. W. Strong and P. Nielaba, JCAP 1605, 056 (2016).

[7] M. Unger and G. R. Farrar, PoS ICRC 2017, 558 (2018). 
[8] M. Unger and G. Farrar, EPJ Web Conf. 210, 04005 (2019).

[9] J. L. Han. R. N. Manchester, W. van Straten, P. Demerest, Astrophys. J. Suppl. 234, 11 (2018).

[10] M. S. Pshirkov, P. G. Tinyakov and F. R. Urban, Mon. Not. Roy. Astron. Soc. 436, 2326 (2013).

[11] G. R. Farrar, Comptes Rendus Physique 15, 339 (2014).

[12] M. S. Pshirkov, P. G. Tinyakov and F. R. Urban, Phys. Rev. Lett. 116, 191302 (2016).

[13] E. Waxman and J. Miralda-Escude, Astrophys. J. 472, L89 (1996)

[14] G. R. Farrar, R. Jansson, I. J. Feain and B. M. Gaensler, JCAP 1301, 023 (2013)

[15] L. A. Anchordoqui, G. E. Romero and J. A. Combi, Phys. Rev. D 60, 103001 (1999).

[16] L. A. Anchordoqui, H. Goldberg and D. F. Torres, Phys. Rev. D 67, 123006 (2003)

[17] R. S. Nemmen, C. Bonatto and T. Storchi-Bergmann, Astrophys. J. 722, 281 (2010)

[18] A. Aab et al. [Pierre Auger Collaboration], arXiv:1708.06592.

[19] A. Aab et al. [Pierre Auger Collaboration], Astrophys. J. 853, no. 2, L29 (2018).

[20] M. Ackermann et al. [Fermi-LAT Collaboration], Astrophys. J. 755, 164 (2012).

[21] K. Greisen, Phys. Rev. Lett. 16, 748 (1966).

[22] G. T. Zatsepin and V. A. Kuzmin, JETP Lett. 4, 78 (1966) [Pisma Zh. Eksp. Teor. Fiz. 4, 114 (1966)].

[23] R. U. Abbasi et al. [Telescope Array Collaboration], Astrophys. J. 867, no. 2, L27 (2018).

[24] R. U. Abbasi et al. [Telescope Array Collaboration], Astrophys. J. 790, L21 (2014).

[25] H. N. He, A. Kusenko, S. Nagataki, B. B. Zhang, R. Z. Yang and Y. Z. Fan, Phys. Rev. D 93, 043011 (2016).

[26] D. N. Pfeffer, E. D. Kovetz and M. Kamionkowski, Mon. Not. Roy. Astron. Soc. 466, 2922 (2017).

[27] J. Biteau et al. [Pierre Auger and Telescope Array Collaborations], EPJ Web Conf. 210, 01005 (2019).

[28] T.-P. Li and Y.-Q. Ma, Astrophys. J. 272, 317 (1983).

[29] L. A. Anchordoqui, Phys. Rev. D 97, no. 6, 063010 (2018).

[30] L. A. Anchordoqui, Phys. Rep. 801, 1 (2019).

[31] L. A. Anchordoqui and J. F. Soriano, arXiv:1905.13243.

[32] S. Mollerach and E. Roulet, Prog. Part. Nucl. Phys. 98, 85 (2018).

[33] G. R. Farrar and M. S. Sutherland, JCAP 1905, 004 (2019).

[34] R. C. dos Anjos et al., Phys. Rev. D 98, 123018 (2018).

[35] A. R. Taylor, J. M. Stil, and C. Sunstrum, Astrophys. J. 702, 1230 (2009).

[36] J. Irwin et al., Astron. J. 144, 43 (2012).

[37] B. Gold et al., Astrophys. J. Suppl. 192, 15 (2011).

[38] C. L. Bennett et al. [WMAP Collaboration], Astrophys. J. Suppl. 208, 20 (2013).

[39] R. Adam et al. [Planck Collaboration], Astron. Astrophys. 594, A10 (2016).

[40] J. F. Soriano, L. A. Anchordoqui and D. F. Torres, Phys. Rev. D 98, 043001 (2018).

[41] A. V. Olinto et al., PoS ICRC 2017, 542 (2018).

[42] L. A. Anchordoqui et al., arXiv:1907.03694.

[43] L. A. Anchordoqui and H. Goldberg, Phys. Rev. D 65, 021302 (2002) 